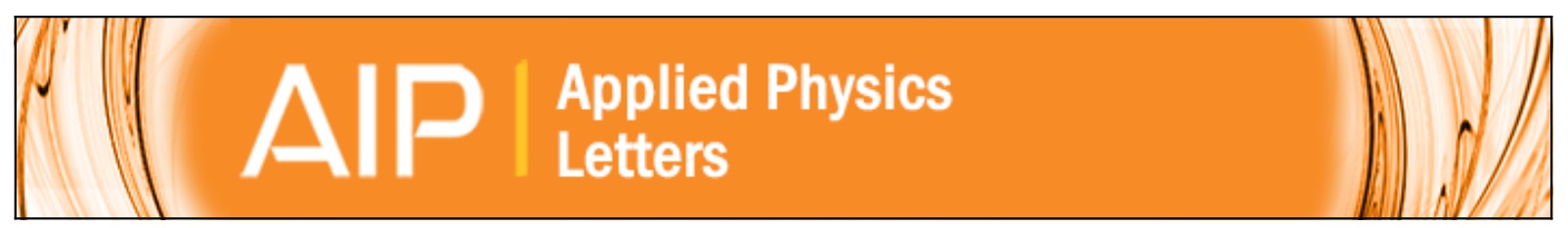

\title{
Substrate effects on quasiparticles and excitons in graphene nanoflakes
}

Weidong Sheng, Mengchao Sun, Aiping Zhou, and S. J. Xu

Citation: Applied Physics Letters 103, 143109 (2013); doi: 10.1063/1.4823829

View online: http://dx.doi.org/10.1063/1.4823829

View Table of Contents: http://scitation.aip.org/content/aip/journal/apl/103/14?ver=pdfcov

Published by the AIP Publishing

\section{Articles you may be interested in}

Energy levels of exciton in a gapped graphene sheet

J. Appl. Phys. 114, 073702 (2013); 10.1063/1.4818613

Exciton effects in boron-nitride (BN) nanotubes

AIP Conf. Proc. 1504, 597 (2012); 10.1063/1.4771767

Quasiparticle energies and uniaxial pressure effects on the properties of $\mathrm{SnO} 2$

Appl. Phys. Lett. 97, 261901 (2010); 10.1063/1.3532109

Toward a theory of the quantum Hall effect in graphene

Low Temp. Phys. 34, 790 (2008); 10.1063/1.2981388

Excitons in $\mathrm{Si} 1 \times \mathrm{Ge} \times$ nanocrystals: Ab initio calculations

J. Appl. Phys. 103, 103716 (2008); 10.1063/1.2913314

\section{Asylum Research Atomic Force Microscopes}

Unmatched Performance, Versatility and Support

Modulus of Polymers

\& Advanced Materials

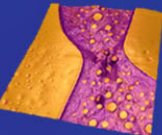

Coating Uniformity \& Roughness

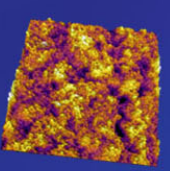

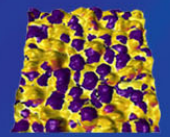

Nanoscale Conductivity \& Permittivity Mapping
Piezoelectrics

\& Ferroelectrics

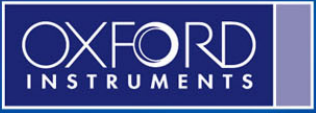

The Business of Science
+1 (805) 696-6466 sales@AsylumResearch.com www.AsylumResearch.com 


\title{
Substrate effects on quasiparticles and excitons in graphene nanoflakes
}

\author{
Weidong Sheng, ${ }^{1, a)}$ Mengchao Sun, ${ }^{1}$ Aiping Zhou, ${ }^{2}$ and S. J. Xu ${ }^{3}$ \\ ${ }^{1}$ State Key Laboratory of Surface Physics and Department of Physics, Fudan University, \\ Shanghai 200433, China \\ ${ }^{2}$ Department of Mathematics and Physics, Nanjing Institute of Technology, Nanjing 211167, China \\ ${ }^{3}$ Department of Physics, The University of Hong Kong, Pokfulam Road, Hong Kong, China
}

(Received 12 July 2013; accepted 17 September 2013; published online 1 October 2013)

\begin{abstract}
The effects of substrate on electronic and optical properties of triangular and hexagonal graphene nanoflakes with armchair edges are investigated by using a configuration interaction approach beyond double excitation scheme. The quasiparticle correction to the energy gap and exciton binding energy are found to be dominated by the long-range Coulomb interactions and exhibit similar dependence on the dielectric constant of the substrate, which leads to a cancellation of their contributions to the optical gap. As a result, the optical gaps are shown to be insensitive to the dielectric environment and unexpectedly close to the single-particle gaps.

(C) 2013 AIP Publishing LLC. [http://dx.doi.org/10.1063/1.4823829]
\end{abstract}

Graphene, an artificial material discovered recently, ${ }^{1}$ is a promising candidate in future microelectronic devices due to its extraordinary electronic ${ }^{2}$ and optical properties. ${ }^{3}$ Recently, many theoretical interests have been attracted to the study of substrate influence on the electronic structure, thermal conductivity, and growth mechanisms in bulk graphene $^{4,5}$ and graphene nanoribbons. ${ }^{6,7}$ Experimentally, the effect of semi-insulating and metal substrates has been investigated by using ultraviolet and far-infrared photoelectron spectroscopy. 8,9

Although bulk graphene has almost zero band-gap, a finite gap can be opened and even engineered by quantum confinement effect in graphene nanoribbons and nanoflakes. ${ }^{10}$ Electron-electron interactions would further modify this quasiparticle gap into the optical gap, which is commonly known as the excitonic effect. ${ }^{11-14}$ Many-body perturbation theory and configuration interaction methods have been applied to calculate exciton binding energies in quasione-dimensional graphene nanoribbons ${ }^{15,16}$ and excitonic absorption in triangular graphene quantum dots with zigzag edges. ${ }^{17,18}$ Undoubtedly, the study of quasiparticle and excitonic effects in these structures requires a proper treatment of the dielectric screening effect ${ }^{19}$ from various substrates like $\mathrm{SiO}_{2},{ }^{20}$ diamond, ${ }^{21} \mathrm{SiC},{ }^{22}$ or other semi-insulating materials.

At present, however, there have been very few attempts to investigate substrate effects on electronic structure and optical properties in graphene nanoflakes. In this letter, we will explore how various substrates affect quasiparticle selfenergies, exciton binding energies, and optical gaps in graphene nanoflakes. An interesting question that how sensitive the optical transitions are to the dielectric environment in nanographene structures, which is believed to have both fundamental and practical importance, will be answered.

We consider two types of armchair graphene nanoflakes placed on various substrates such as $\mathrm{SiO}_{2}$, diamond, and $\mathrm{SiC}$. Figure 1 gives a schematic view of our first model system, a triangular graphene nanoflake. The number of carbon rings

a)Email: shengw@fudan.edu.cn along each edge is set to be $N=4$, which corresponds to a total number of atoms $n=60$. The single-particle states are obtained by the use of the tight-binding model with the nearest-neighbor hopping. The matrix element of the single-particle Hamiltonian for electron $p$ is given by $\langle i|\hat{\mathbf{H}}(p)| j\rangle=t$, if site $i$ and $j$ are the nearest neighbors and would vanish if otherwise. The hopping energy is set to be $-2.7 \mathrm{eV}$. The single-particle states, $\psi_{m}=\sum_{i=1}^{N} c_{m}^{i}|i\rangle$, are calculated by diagonalizing the Hamiltonian matrix and are plotted in Fig. 1. A single-particle gap is seen to separate the occupied and unoccupied states. Moreover, the singleparticle energies are found not continuous; instead, the energy levels form a series of clusters.

As electron-electron interactions exhibit different dimensional dependence in graphene from other semiconductors, ${ }^{23}$ we make use of configuration interaction method to solve the interacting electron problem. Many-particle wave functions are expanded on the basis of single-particle states obtained previously by the tight-binding method. Unlike those structures with zigzag edges,${ }^{24}$ the nanoflake with armchair edges is seen to have a closed-shell energy spectrum with a well-defined energy gap. Therefore, we have to choose a number of valence states $\left(N_{s}\right)$ from the HOMO (highest occupied molecular orbital) down and the same number of conduction states from LUMO (lowest unoccupied molecular orbital) up, as our basis to expand the following many-particle Hamiltonian:

$$
\begin{aligned}
\hat{\mathbf{H}} & =\sum_{p=1}^{N_{e}} \hat{\mathbf{H}}(p)+\frac{1}{\epsilon_{r}^{*}} \sum_{p \neq q}^{N_{e}} \hat{\mathbf{V}}(p, q), \\
\hat{\mathbf{V}}(p, q) & =\frac{e^{2}}{4 \pi \epsilon_{0} \sqrt{\left|x_{p}-x_{q}\right|^{2}+\left|y_{p}-y_{q}\right|^{2}}},
\end{aligned}
$$

where $N_{e}$ is the number of electrons which equals to $N_{s}$ in our neutral half-filling system. As single-particle energy levels form a series of clusters, we choose the $N_{s}$-th level as the end of a cluster of states to ensure that $E_{N_{s}+1}-E_{N_{s}}$ is large 


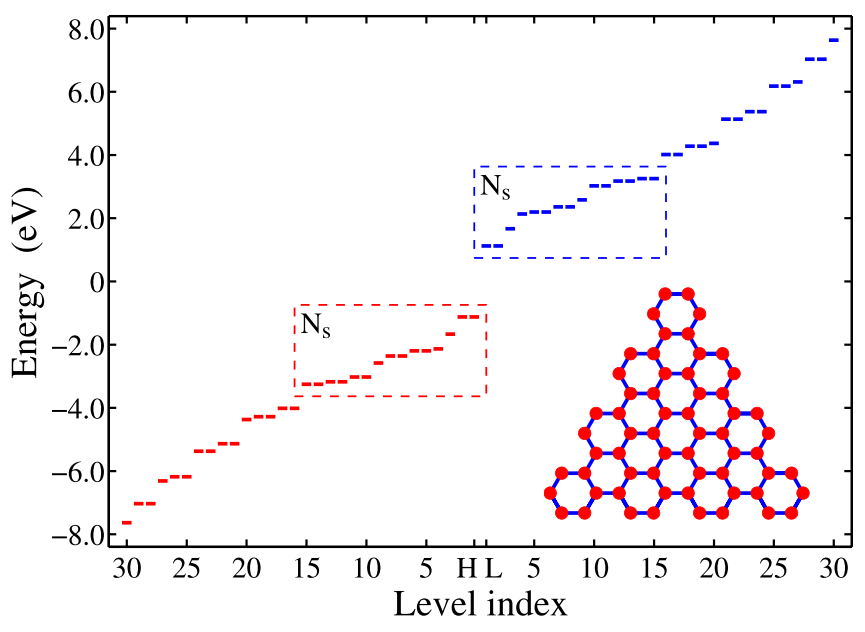

FIG. 1. Energy levels of a triangular graphene nanoflake (as shown in the inset). The HOMO and LUMO states are denoted by $H$ and $L$, respectively. The number of electron (hole) states (schematically shown in two dashed boxes) taken into account in the configuration interaction computation is denoted by $N_{s}$.

enough compared with the pertinent Coulomb energies. Here we set $N_{s}=16$ and have $E_{N_{s}+1}-E_{N_{s}}=0.22 \mathrm{eV}$. For an electron excited from the $N_{s^{-}}$to $\left(N_{s}+1\right)$-th state, the Coulomb energy decreases by $0.074 \mathrm{eV}$, which is much smaller than the increment of the kinetic energy $0.22 \mathrm{eV}$.

The effective background dielectric constant is determined by $\epsilon_{r}^{*}=\frac{1}{2}\left(\epsilon_{r}+1\right)$ with $\epsilon_{r}$ for the substrate. ${ }^{25}$ For $\mathrm{SiO}_{2}$, diamond, and $\mathrm{SiC}, \epsilon_{r}^{*}$ is given by $2.5,3.35$, and 6.4 , respectively. The Coulomb matrix elements ${ }^{26}$ consist of the on-site and off-site parts as follows:

$$
U_{p q r s}=\sum_{i=1}^{N} c_{p}^{i} c_{q}^{i} U_{00} c_{r}^{i} c_{s}^{i}+\frac{1}{\epsilon_{r}^{*}} \sum_{i \neq j}^{N} c_{p}^{i} c_{q}^{j} \hat{\mathbf{V}}(i, j) c_{r}^{i} c_{s}^{j},
$$

in which $U_{00}=17.0 \mathrm{eV}$ is chosen for the on-site Coulomb interaction. ${ }^{27}$ It is noted that only the off-site part is influenced by the dielectric screening. All the occupied states in the closed-shell system form a single reference configuration. For a given $N_{s}$, one can choose to move $m\left(\leq N_{s}\right)$ electrons from the occupied states to the unoccupied states, usually referred as a $m$-th excitation, to construct a manyparticle configuration. For the model systems considered in this work, we find that it is necessary to have $m \geq 5$ in order for the low-lying levels to be fully converged (see details below). Here, we choose $\left(N_{s}, m\right)=(16,5)$, and the resulting sparse matrix has a dimension of $6,689,001$. ARPACK is used for the diagonalization of the matrix to obtain the energy levels $E_{n}\left(N_{e}\right)$ of the many-electron system.

For a given occupation number $N_{e}$, the quasiparticle gap can be then obtained by

$$
E_{\text {gap }}^{q p}=\mu\left(N_{e}+1\right)-\mu\left(N_{e}\right)
$$

where $\mu\left(N_{e}\right)$ and $\mu\left(N_{e}+1\right)$ are the chemical potentials of the system defined by

$$
\begin{aligned}
\mu\left(N_{e}\right) & =E_{0}\left(N_{e}\right)-E_{0}\left(N_{e}-1\right), \\
\mu\left(N_{e}+1\right) & =E_{0}\left(N_{e}+1\right)-E_{0}\left(N_{e}\right),
\end{aligned}
$$

with $E_{0}\left(N_{e}\right)$ being the ground-state energy of the $N_{e}$-electron system. It is noted that the basis dimension of the system with either an extra electron $\left(N_{e}+1\right)$ or hole $\left(N_{e}-1\right)$ increases by almost twice. The excitonic or optical $\operatorname{gap}^{28}$ is defined by

$$
E_{g a p}^{o p}=E_{1}^{S=0}\left(N_{e}\right)-E_{0}^{S=0}\left(N_{e}\right) .
$$

The quasiparticle and optical gap is related by the exciton binding energy $E_{X}$ as follows:

$$
E_{X}=E_{g a p}^{q p}-E_{g a p}^{o p} .
$$

Table I lists the quasiparticle gap $E_{g a p}^{q p}$ and quasiparticle correction to the energy gap $E_{g a p}^{q p}-E_{g a p}^{s p}$ calculated for various substrates. First, we would like to mention that the chemical potential we calculate is only for the interacting electron system because the background ionic charges only shifts all addition energies in the same way and thus shall have little effect on the quasiparticle gap. Compared with the singleparticle gap $E_{g a p}^{s p} \approx 2.25 \mathrm{eV}$, we see that the quasiparticle gaps are larger by about $0.99-1.58 \mathrm{eV}$ due to strong electronelectron interactions. The Coulomb matrix elements averaged among the HOMO and LUMO states are found to be $1.47 \mathrm{eV}$ (direct) and $0.35 \mathrm{eV}$ (exchange), which is either larger than or comparable with the corresponding kinetic energy $E_{g a p}^{s p} / 2=1.12 \mathrm{eV}$. Moreover, a typical correlation element is found to be about one third of the exchange term and thus would make a non-negligible contribution to the total energy. In the case of $\mathrm{SiC}$ substrate, the ground-state energy obtained by the configuration-interaction method is $35.18 \mathrm{eV}$ while an unrestricted Hartree-Fock calculation gives $37.14 \mathrm{eV}$. The difference, i.e., the correlation energy is therefore seen to account for about $1.96 \mathrm{eV}$.

As the substrate changes from $\mathrm{SiC}$ to $\mathrm{SiO}_{2}$, we find that the quasiparticle self-energy correction to the energy gap, i.e., $E_{\text {gap }}^{q p}-E_{\text {gap }}^{\text {sp }}$, increases by about $60 \%$ or from $0.99 \mathrm{eV}$ to $1.58 \mathrm{eV}$. Considering that this increment occurs as a result of the reduction of the effective dielectric constant also by $60 \%$, we believe that the quasiparticle effect is dominated by the long-range Coulomb interaction which is controlled by $\epsilon_{r}^{*}$. Actually, if one removes the long-range Coulomb interaction by setting $\epsilon_{r}^{*} \rightarrow \infty, E_{g a p}^{q p}-E_{g a p}^{s p}$ would reduce to $0.49 \mathrm{eV}$. In the case of $\mathrm{SiO}_{2}$ substrate, this means that the onsite Coulomb interaction contributes only about $30 \%$ of the overall quasiparticle effect.

Figure 2 plots the energy spectra for the triangular model for three different substrates. Above the singlet ground state, we see three triplet $(S=1)$ states before the first excited state of $S=0$. The calculated optical gap is plotted in open dots as a function of the effective dielectric constant. As a reference,

TABLE I. List of quasiparticle gap and quasiparticle correction to the energy gap calculated for various substrates.

\begin{tabular}{lccc}
\hline \hline Substrate & $\epsilon_{r}^{*}$ & $E_{g a p}^{q p}$ & $E_{g a p}^{q p}-E_{g a p}^{s p}$ \\
\hline Silicon carbide & 6.4 & 3.2384 & 0.9930 \\
Diamond & 3.35 & 3.5911 & 1.3457 \\
Silicon dioxide & 2.5 & 3.8257 & 1.5803 \\
\hline
\end{tabular}




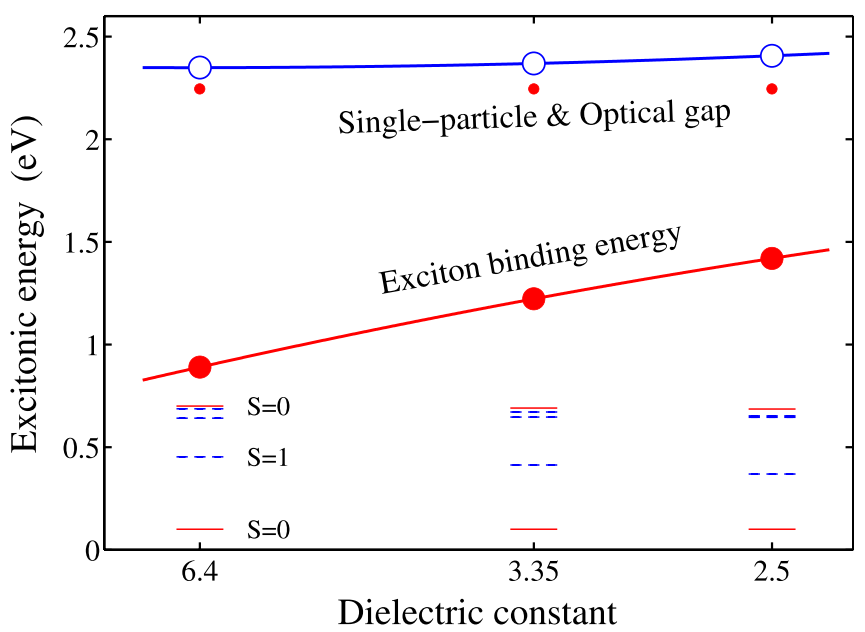

FIG. 2. Optical gap (in open dots) and exciton binding energy (in solid dots) calculated as a function of the inverse of the effective dielectric constant. The single-particle gap, which does not vary with the dielectric constant, is shown in the smaller solid dots as a reference. Insets: Schematic view of the energy levels and their total spins. Solid lines for spin singlets and dashed lines for spin triplets.

the single-particle gap is shown in solid dots just below the optical gaps. For the $\mathrm{SiO}_{2}$ substrate, we have $E_{g a p}^{o p}=2.41 \mathrm{eV}$. It is noted that the double excitation scheme $(m=2)$ gives a value of $3.38 \mathrm{eV}$ which is far from convergence. We find that the satisfied convergence can only be achieved until $m=5$ as the calculation shows that $E_{\text {gap }}^{o p}=2.51 \mathrm{eV}$ for $m=4$ and $2.40 \mathrm{eV}$ for $m=6$.

When the substrate changes from $\mathrm{SiC}$ to $\mathrm{SiO}_{2}$, the relative difference between $E_{g a p}^{o p}$ and $E_{g a p}^{s p}$ is found to increase from $0.1 \mathrm{eV}$ to $0.16 \mathrm{eV}$. However, this difference is so small that the optical gap is close to the single-particle gap and its absolute value increases by only $2.5 \%$. Compared with the quasiparticle gap, the substrate hence plays only a minor role in the optical gap. In other words, the optical gap is insensitive to the long-range Coulomb interactions. Then how about short-range interactions? As the on-site Coulomb interaction $U_{00}$ reduces from $17.0 \mathrm{eV}$ to $9.3 \mathrm{eV}$, we find that the optical gap decreases from $2.41 \mathrm{eV}$ to $2.39 \mathrm{eV}$ by less than $1 \%$. Therefore, we can safely conclude that the optical gap is sensitive to neither the long-range nor short-range Coulomb interactions. In fact, we see that the two gaps $E_{g a p}^{o p}$ and $E_{g a p}^{s p}$ differ from each other by less than $5 \%$ in the case of SiC substrate.

To see why the optical gap is insensitive to both the long-range and short-range Coulomb interactions, we plot the exciton binding energy in Fig. 2 . It is found that $E_{X}$ increases from about $0.89 \mathrm{eV}$ to $1.42 \mathrm{eV}$ as the substrate changes from $\mathrm{SiC}$ to $\mathrm{SiO}_{2}$. This range happens to be similar to the previous first-principles calculations on graphene nanoribbons. ${ }^{28}$ It is reminded that $E_{g a p}^{q p}$ gains about $0.59 \mathrm{eV}$ when $\epsilon_{r}^{*}$ decreases 6.4-2.5. In the meantime, due to the same substrate change, $E_{X}$ increases by about $0.53 \mathrm{eV}$. Considering that the quasiparticle gap and exciton binding energy contribute to the optical gap in the opposite way, i.e., $E_{g a p}^{o p}=E_{g a p}^{q p}-E_{X}$, the net change in the optical gap is only $0.59-0.53=0.06 \mathrm{eV}$, one order of magnitude smaller than either $E_{g a p}^{q p}$ or $E_{X}$. The Coulombic energy $E_{X}$ consists mainly of a polarization contribution while the quasiparticle gap is largely determined by the self-energy contribution. Although both terms are shown to depend strongly on the dielectric environment, what is most interesting here is that the quasiparticle and excitonic effects have very similar dependence on the dielectric constant. In fact, we find that the exciton effect is also dominated by the long-range Coulomb interaction. The on-site Coulomb interaction gives an exciton binding energy of $0.38 \mathrm{eV}$, which is less than $30 \%$ of the overall exciton binding energy in the case of $\mathrm{SiO}_{2}$ substrate.

Our next model system is a hexagonal graphene nanoflake with armchair edges, which has a similar singleparticle spectrum to the previous triangular model. Figure 3 plots the quasiparticle and optical gaps together with the exciton binding energy as a function of the inverse of dielectric constant. Overall, we find that $E_{g a p}^{q p}, E_{g a p}^{o p}$, and $E_{X}$ exhibit very similar dependence on $\epsilon_{r}^{*}$ to those seen for the triangular model. Specifically, as the substrate changes from $\mathrm{SiC}$ to $\mathrm{SiO}_{2}$, the quasiparticle correction to the energy gap is seen to increase from $0.6 \mathrm{eV}$ to $1.06 \mathrm{eV}$ by about $0.46 \mathrm{eV}$ while the exciton binding energy from $0.59 \mathrm{eV}$ to $0.93 \mathrm{eV}$ by $0.34 \mathrm{eV}$. As a result, the optical gap gains about $0.12 \mathrm{eV}$ due to the reduced screening effect. Furthermore, most noticeably is that the optical gap becomes almost identical to the singleparticle gap in the case of $\mathrm{SiC}$ substrate.

Figure 4 shows the size dependence of the singleparticle and optical gaps, quasiparticle correction to the energy gap and exciton binding energy for the hexagonal nanoflake on a $\mathrm{SiC}$ substrate. When the size increases, the quantum confinement looses its effect and all the gaps as well as the exciton binding energy are seen to gradually decrease. As for the quasiparticle effect, let us reformulate Eq. (6) as follows:

$$
E_{\text {gap }}^{o p}-E_{\text {gap }}^{s p}=\left(E_{\text {gap }}^{q p}-E_{\text {gap }}^{s p}\right)-E_{X} .
$$

As the size increases, the quasiparticle correction to the energy gap, i.e., $E_{g a p}^{q p}-E_{g a p}^{s p}$ and the exciton binding energy $E_{X}$ are found to exhibit nearly the same dimensional dependence, which leads to an almost exact cancellation of their

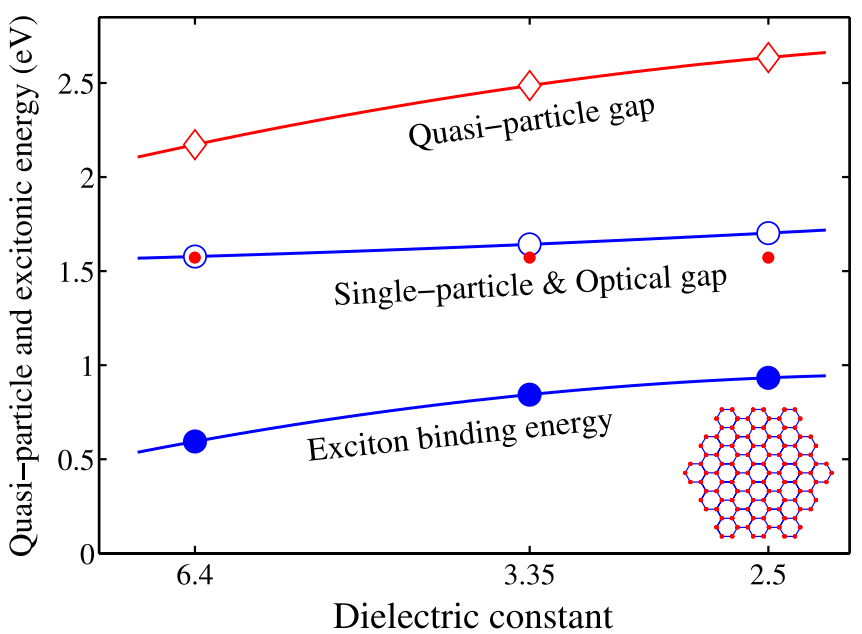

FIG. 3. For a hexagonal nanoflake with $N=3$ as shown in the inset, quasiparticle (diamonds) and optical gap (open dots) as well as the exciton binding energy (solid dots) as a function of the inverse of the effective dielectric constant. The single-particle gap, which does not vary with the dielectric constant, is shown in the smaller solid dots as a reference. 


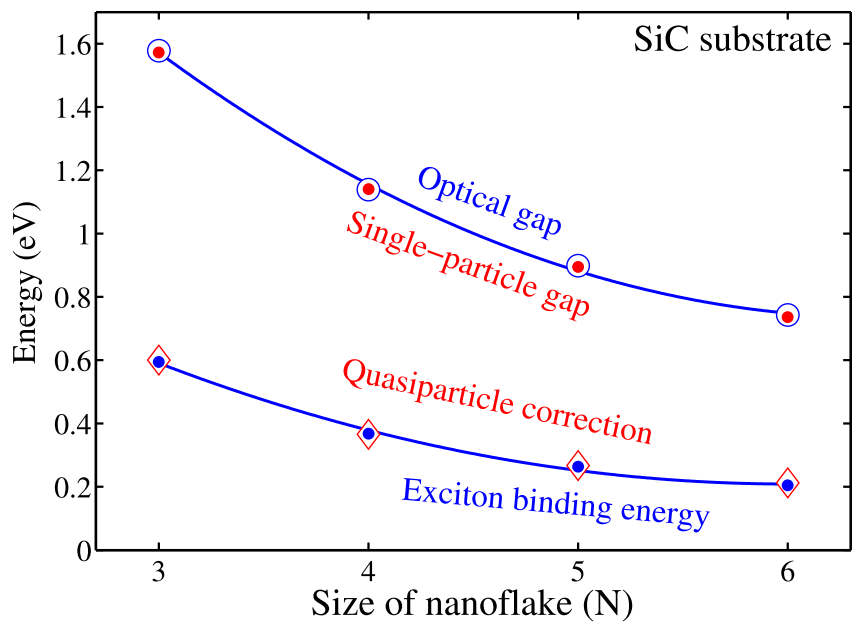

FIG. 4. Single-particle gap (solid dots), quasiparticle correction (diamonds), optical gap (open dots), and exciton binding energy (solid dots) calculated as a function of the size of the hexagonal nanoflake on a SiC substrate.

contributions to the optical gap. As a result, we see that $E_{\text {gap }}^{o p}-E_{\text {gap }}^{s p}$ nearly vanishes, i.e., the optical gap closely follows the single-particle gap. The surprising overlap of the single-particle and optical gaps can be explained in the following. We have seen that a small difference between the quasiparticle correction and exciton binding energy is caused by the long-range Coulomb interaction. In the case of $\mathrm{SiC}$ substrate where the long-range Coulomb interaction is greatly suppressed, both $E_{g a p}^{q p}-E_{g a p}^{s p}$ and $E_{X}$ are now mainly determined by the short-range Coulomb interaction and thus become almost identical to each other.

In summary, we have carried out a configurationinteraction study of quasiparticle and excitonic effects in graphene nanoflakes on various substrates. We have identified that both the quasiparticle correction to the energy gap and exciton binding energy are dominated by the long-range Coulomb interactions, and furthermore, these two terms exhibit similar dependence on the dielectric constant of the substrate. As a result, their contributions to the optical gap almost cancel each other, which leads to a weak dependence of the optical gap on the dielectric environment. In the case of substrate with larger dielectric constant, and thus strong screening effect like $\mathrm{SiC}$, the optical gaps of graphene nanoflakes are revealed to closely follow the single-particle gap as if all the electron-electron interactions are quenched.

This work was supported by National Basic Research Program of China (973 Program No. 2011CB925602). Xu acknowledges the support by Shenzhen Basic Research Grants (No. JCYJ20120615142933076).

${ }^{1}$ K. S. Novoselov, A. K. Geim, S. V. Morozov, D. Jiang, Y. Zhang, S. V. Dubonos, I. V. Grigorieva, and A. A. Firsov, Science 306, 666 (2004).

${ }^{2}$ S. Y. Zhou, G.-H. Gweon, J. Graf, A. V. Fedorov, C. D. Spataru, R. D. Diehl, Y. Kopelevich, D.-H. Lee, S. G. Louie, and A. Lanzara, Nat. Phys. 2, 595 (2006).

${ }^{3}$ T. Mueller, F. Xia, and P. Avouris, Nat. Photonics 4, 297 (2010).

${ }^{4}$ X. Li, B. D. Kong, J. M. Zavada, and K. W. Kim, Appl. Phys. Lett. 99, 233114 (2011).

${ }^{5}$ V. O. Özçelik, S. Cahangirov, and S. Ciraci, Phys. Rev. B 85, 235456 (2012). ${ }^{6}$ L. Liang and V. Meunier, Phys. Rev. B 86, 195404 (2012).

${ }^{7}$ J. Chen, M. Vanin, Y. Hu, and H. Guo, Phys. Rev. B 86, 075146 (2012).

${ }^{8}$ Q.-H. Wu, G. Hong, T. W. Ng, and S. T. Lee, Appl. Phys. Lett. 100, 161603 (2012).

${ }^{9}$ J. Y. Kim, C. Lee, S. Bae, K. S. Kim, B. H. Hong, and E. J. Choi, Appl. Phys. Lett. 98, 201907 (2011).

${ }^{10}$ Y.-W. Son, M. L. Cohen, and S. G. Louie, Phys. Rev. Lett. 97, 216803 (2006).

${ }^{11}$ R. Dillenschneider and J. H. Han, Phys. Rev. B 78, 045401 (2008).

${ }^{12}$ R. Nandkishore and L. Levitov, Phys. Rev. Lett. 104, 156803 (2010).

${ }^{13}$ J. Sabio, F. Sols, and F. Guinea, Phys. Rev. B 82, 121413(R) (2010).

${ }^{14}$ T. Paananen and R. Egger, Phys. Rev. B 84, 155456 (2011).

${ }^{15}$ L. Yang, C.-H. Park, Y.-W. Son, M. L. Cohen, and S. G. Louie, Phys. Rev. Lett. 99, 186801 (2007).

${ }^{16}$ G. Luo, X. Qian, H. Liu, R. Qin, J. Zhou, L. Li, Z. Gao, E. Wang, W.-N. Mei, J. Lu, Y. Li, and S. Nagase, Phys. Rev. B 84, 075439 (2011).

${ }^{17}$ S. Dutta, S. Lakshmi, and S. K. Pati, Phys. Rev. B 77, 073412 (2008).

${ }^{18}$ A. D. Güçlü, P. Potasz, and P. Hawrylak, Phys. Rev. B 82, 155445 (2010).

${ }^{19}$ C. Jang, S. Adam, J.-H. Chen, E. D. Williams, S. D. Sarma, and M. S. Fuhrer, Phys. Rev. Lett. 101, 146805 (2008).

${ }^{20}$ M. Ishigami, J. H. Chen, W. G. Cullen, M. S. Fuhrer, and E. D. Williams, Nano Lett. 7, 1643 (2007).

${ }^{21}$ Y. Wu, Y.-M. Lin, A. A. Bol, K. A. Jenkins, F. Xia, D. B. Farmer, Y. Zhu, and P. Avouris, Nature 472, 74 (2011).

${ }^{22}$ W. Strupinski, K. Grodecki, A. Wysmolek, R. Stepniewski, T. Szkopek, P. E. Gaskell, A. Grüneis, D. Haberer, R. Bozek, J. Krupka, and J. M. Baranowski, Nano Lett. 11, 1786 (2011).

${ }^{23}$ V. N. Kotov, B. Uchoa, V. M. Pereira, F. Guinea, and A. H. Castro Neto, Rev. Mod. Phys. 84, 1067 (2012).

${ }^{24}$ W. Sheng, M. Sun, and A. Zhou, Phys. Rev. B 88, 085432 (2013).

${ }^{25}$ D. A. Siegel, C.-H. Park, C. Hwang, J. Deslippe, A. V. Fedorov, S. G. Louie, and A. Lanzara, Proc. Natl. Acad. Sci. U.S.A. 108, 11365 (2011).

${ }^{26}$ W. Sheng, S.-J. Cheng, and P. Hawrylak, Phys. Rev. B 71, 035316 (2005).

${ }^{27}$ T. O. Wehling, E. Şaşioğlu, C. Friedrich, A. I. Lichtenstein, M. I. Katsnelson, and S. Blügel, Phys. Rev. Lett. 106, 236805 (2011).

${ }^{28}$ L. Yang, M. L. Cohen, and S. G. Louie, Nano Lett. 7, 3112 (2007). 